December 16, 2002

LBNL-51881

SLAC-PUB-9611

hep-ph/0212228

\title{
Collider Tests of the Little Higgs Model
}

\author{
Gustavo Burdman and Maxim Perelstein \\ Theoretical Physics Group \\ Ernest Orlando Lawrence Berkeley National Laboratory \\ University of California, Berkeley, California 94720, USA \\ Aaron Pierce \\ Theoretical Physics Group \\ Stanford Linear Accelerator Center \\ Stanford University, Stanford, California,94309, USA
}

\begin{abstract}
The little Higgs model provides an alternative to traditional candidates for new physics at the $\mathrm{TeV}$ scale. The new heavy gauge bosons predicted by this model should be observable at the Large Hadron Collider (LHC). We discuss how the LHC experiments could test the little Higgs model by studying the production and decay of these particles.
\end{abstract}

*The work of GB and MP was supported by the Director, Office of Science, Office of High Energy and Nuclear Physics,U.S. Department of Energy under Contract DE-AC0376SF00098, and in part by the National Science Foundation under grant PHY-0098840. The work of AP was supported by the Director, Office of Science, Office of High Energy and Nuclear Physics,U.S. Department of Energy under Contract DE-AC03-76SF00515 


\section{Introduction}

The hierarchy problem, the question of the origin and radiative stability of the enormous hierarchy between the electroweak symmetry breaking scale and the fundamental scale of gravity, requires new physical phenomena to appear at energy scales around $\mathrm{TeV}$. Several models of new physics at the $\mathrm{TeV}$ scale have been proposed over the years, relying on supersymmetry, new strong dynamics, or extra dimensions to resolve the hierarchy problem. The experiments at the upcoming Large Hadron Collider (LHC) are expected to determine which of these possibilities is realized in Nature.

All models of $\mathrm{TeV}$-scale physics are strongly constrained by non-observation of virtual effects of new particles in experiments at presently available energies. For example, supersymmetric theories require non-trivial model building to satisfy constraints from flavor-changing neutral currents (FCNC). Recently, an elegant and general way to avoid such indirect constraints has been suggested [1, 2]. In this proposal, the hierarchy problem is solved in two steps. First, the Standard Model (SM) in incorporated into a "little Higgs" theory that possesses enough symmetry to cancel the quadratically divergent contribution to the Higgs mass parameter at one-loop order. This cancellation allows the little Higgs theory to be valid up to a scale of order 10 $\mathrm{TeV}$ without any fine tuning. Around $10 \mathrm{TeV}$, the little Higgs theory itself breaks down, and the full dynamics resolving the hierarchy problem becomes apparent. This dynamics may be described by one of the traditional solutions of the hierarchy problem, e.g. a supersymmetric theory. The simple structure of the little Higgs theory, combined with the high energy scale at which the full set of new particles and interactions become relevant, implies that this scenario can easily satisfy indirect constraints such as FCNC.

The little Higgs models necessarily contain new particles whose virtual contributions cancel the one-loop quadratic divergence in the Higgs mass parameter. To avoid fine tuning, these particles should not be much heavier than $1 \mathrm{TeV}$; thus, they are expected to be observable at the LHC. Crucially, the couplings of these particles to the SM Higgs are firmly predicted: indeed, the cancellation of quadratic divergences can only occur for particular values of these couplings. In this letter, we will discuss how the LHC experiments can not only discover the new states predicted by the little Higgs models, but also verify the structure of the model by measuring their couplings. 


\section{The Model}

We will concentrate on the so-called "littlest Higgs" model 11, based on a non-linear $\sigma$ model describing $S U(5) \rightarrow S O(5)$ symmetry breaking. The symmetry-breaking vacuum expectation value (vev) is proportional to

$$
\Sigma_{0}=\left(\begin{array}{lll}
0 & 0 & \mathbb{1} \\
0 & 1 & 0 \\
\mathbb{1} & 0 & 0
\end{array}\right)
$$

where $\mathbb{1}$ is a $2 \times 2$ identity matrix. The low-energy dynamics is described in terms of the non-linear sigma model field

$$
\Sigma(x)=e^{i \Pi / f} \Sigma_{0} e^{i \Pi^{T} / f}=e^{2 i \Pi / f} \Sigma_{0},
$$

where $f \sim 1 \mathrm{TeV}$ is the decay constant, and $\Pi=\sum_{a} \pi^{a}(x) X^{a}$. The sum runs over the 14 broken $S U(5)$ generators $X^{a}$, and $\pi^{a}(x)$ are the Goldstone bosons. Furthermore, the $[S U(2) \times U(1)]^{2}$ subgroup of the $S U(5)$ symmetry is gauged; the gauged generators are given by

$$
Q_{1}^{a}=\left(\begin{array}{c}
\sigma^{a} / 2 \\
\end{array}\right), \quad Q_{2}^{a}=\left(\begin{array}{c} 
\\
-\sigma^{a *} / 2
\end{array}\right)
$$

for the $S U(2)$ factors. We write the $U(1)$ factors as $Y_{1}=\operatorname{diag}(-3,-3,2,2,2) / 10$ and $Y_{2}=\operatorname{diag}(-2,-2,-2,3,3) / 10$. The gauge kinetic term has the form

$$
\frac{f^{2}}{8} \operatorname{Tr}\left(D_{\mu} \Sigma\right)\left(D^{\mu} \Sigma\right)^{\dagger}
$$

The covariant derivative of the $\Sigma$ field is given by

$$
\begin{aligned}
D_{\mu} \Sigma=\partial_{\mu} \Sigma- & i \sum_{j=1}^{2} g_{j} W_{j}^{a}\left(Q_{j}^{a} \Sigma+\Sigma Q_{j}^{a T}\right)- \\
& i \sum_{j=1}^{2} g_{j}^{\prime} B_{j}\left(Y_{j} \Sigma+\Sigma Y_{j}\right),
\end{aligned}
$$

where $W_{j}^{a}(a=1 \ldots 3)$ and $B_{j}$ are the $S U(2)$ and $U(1)$ gauge fields, respectively, and $g_{j}$ and $g_{j}^{\prime}$ are the corresponding gauge couplings. 
The vev (1) breaks the gauge group down to the diagonal $S U(2) \times U(1)$, identified with the SM electroweak group. The linear combinations of the gauge bosons that remain massless are given by $W_{L}^{a}=\sin \psi W_{1}^{a}+\cos \psi W_{2}^{a}$ and $B_{L}=\sin \psi^{\prime} B_{1}+\cos \psi^{\prime} B_{2}$, where the mixing angles are determined by

$$
\tan \psi=g_{2} / g_{1}, \quad \tan \psi^{\prime}=g_{2}^{\prime} / g_{1}^{\prime} \text {. }
$$

The SM $S U(2)_{L}$ and $U(1)_{Y}$ gauge couplings are given by $g=g_{1} \sin \psi$ and $g^{\prime}=g_{1}^{\prime} \sin \psi^{\prime}$, respectively. The orthogonal linear combinations of the gauge bosons, $W_{H}^{a}$ and $B_{H}$, acquire masses

$$
M\left(W_{H}^{a}\right)=\frac{g}{\sin 2 \psi} f, \quad M\left(B_{H}\right)=\frac{g^{\prime}}{\sqrt{5} \sin 2 \psi^{\prime}} f .
$$

Out of the 14 Goldstone bosons of the $S U(5) / S O(5)$ sigma model, four are eaten by $W_{H}^{a}$ and $B_{H}$; the Goldstone boson matrix for the remaining 10 fields has the form

$$
\Pi=\left(\begin{array}{ccc} 
& \frac{H^{\dagger}}{\sqrt{2}} & \phi^{\dagger} \\
\frac{H}{\sqrt{2}} & & \frac{H^{*}}{\sqrt{2}} \\
\phi & \frac{H^{T}}{\sqrt{2}} &
\end{array}\right),
$$

where $H=\left(h^{+}, h_{0}\right)$ is the SM Higgs doublet, and $\phi$ is an additional Higgs field transforming as a triplet under the $\mathrm{SM} S U(2)_{L}$. At tree level, both $H$ and $\phi$ are massless. The couplings of the $H$ field to the gauge bosons follow from (41), and at leading order in $1 / f$ are given by

$$
\frac{1}{4} H\left(g_{1} g_{2} W_{1}^{\mu a} W_{2 \mu}^{a}+g_{1}^{\prime} g_{2}^{\prime} B_{1}^{\mu} B_{2 \mu}\right) H^{\dagger} .
$$

In a generic theory, one would also expect diagonal couplings such as $g_{1}^{2} W_{1}^{2} H H^{\dagger}$ and $g_{2}^{2} W_{2}^{2} H_{H^{\dagger}}$. These couplings generally lead to quadratically divergent renormalization of the Higgs mass parameter. The structure of Eq. (9), in which the diagonal couplings are absent, guarantees the absence of such divergences, and is therefore a crucial feature of the little Higgs model. In terms of the gauge boson mass eigenstates, Eq. (9) reads,

$$
\begin{gathered}
\frac{1}{4} H\left(g^{2}\left(W_{L \mu}^{a} W_{L}^{\mu a}-W_{H \mu}^{a} W_{H}^{\mu a}-2 \cot 2 \psi W_{H \mu}^{a} W_{L}^{\mu a}\right)+\right. \\
\left.g^{\prime 2}\left(B_{L \mu} B_{L}^{\mu}-B_{H \mu} B_{H}^{\mu}-2 \cot 2 \psi^{\prime} B_{H \mu} B_{L}^{\mu}\right)\right) H^{\dagger}
\end{gathered}
$$


In this basis, the diagonal couplings of the Higgs to the gauge bosons are present, but the couplings to the light and heavy states have equal magnitude and opposite sign, ensuring the cancellation of quadratic divergences. There is no such cancellation for the triplet $\phi$, which acquires a mass of order $\mathrm{TeV}$.

Spontaneous electroweak symmetry breaking (EWSB) arises due to the logarithmically divergent negative contribution to the Higgs mass-squared

from top loops [1]. As usual, we parametrize $H=U(x) \frac{1}{\sqrt{2}}(0, v+h(x))$, and choose the unitary gauge, $U(x)=1$. The ratio of the Higgs vev and the decay constant $f$ provides a useful expansion parameter: $\epsilon=v / f \lesssim 0.2$. The triplet $\phi$ is generally also expected to acquire a small vev, $v^{\prime} \ll v[3]$; we will neglect this effect in our analysis.

\section{Higgs-gauge boson couplings}

EWSB induces cubic couplings between the physical Higgs boson and the gauge bosons of the model. The couplings can be obtained from Eq. (41). Consider first the neutral gauge bosons. Let $V=\left(W_{L}^{3}, B_{L}, W_{H}^{3}, B_{H}\right)$; the relevant terms in (44) can be written as

$$
\frac{1}{2} f^{2} V^{\mu}\left(\mathcal{M}_{1}+\left(\epsilon+\frac{h(x)}{f}\right)^{2} \mathcal{M}_{2}+\ldots\right) V_{\mu}^{T},
$$

where $\mathcal{M}_{1}=\operatorname{diag}\left(0,0, g^{2} / \sin ^{2} 2 \psi, g^{\prime 2} / 5 \sin ^{2} 2 \psi^{\prime}\right)$, and the dots represent terms of higher order in $1 / f$. The gauge boson mass matrix to second order in $\epsilon$, $\mathcal{M}_{1}+\epsilon^{2} \mathcal{M}_{2}$, is not diagonal in the chosen basis; to diagonalize it, we perform the standard rotation by the Weinberg angle $\theta_{\mathrm{W}}$ in the upper left corner taking $\left(W_{L}^{3}, B_{L}\right) \rightarrow(A, Z)$. 

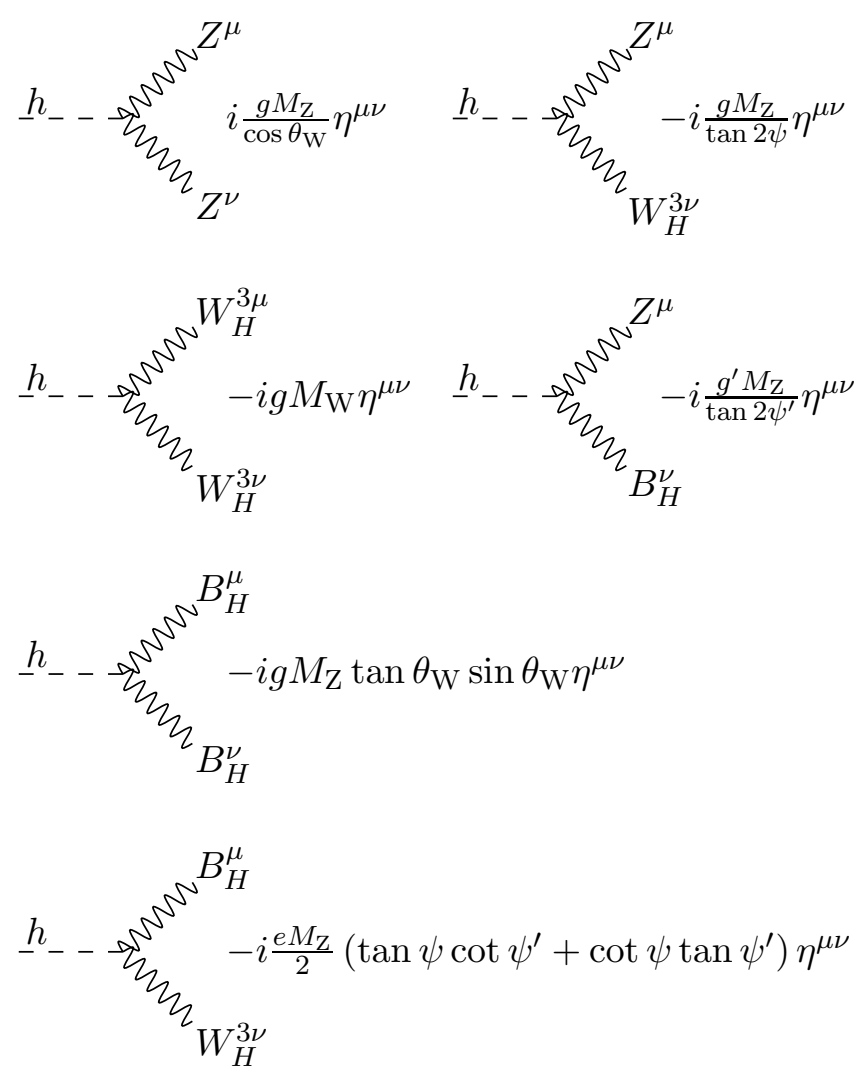

Figure 1: Cubic couplings between the physical Higgs boson $h$ and pairs of neutral gauge bosons.

All other rotations required to diagonalize the mass matrix involve angles of order $\epsilon$; we will not need to know them explicitly. The Higgs couplings to pairs of gauge bosons are proportional to the elements of the matrix $\mathcal{M}_{2}$; we collect the non-vanishing couplings in Fig. 1, keeping only the leading order in $\epsilon$. Note that $\mathcal{M}_{2}$ is not diagonal in the mass eigenbasis, leading to off-diagonal Higgs couplings such as $W_{H}^{3} Z h$. The cubic couplings of the Higgs to charged gauge bosons are computed in the same fashion; they are shown in Fig. 2.

The three diagonal couplings of the Higgs in Fig. $1, h Z Z, h W_{H}^{3} W_{H}^{3}$ and $h B_{H} B_{H}$, add up to zero. So do the two diagonal couplings in Fig. $2, h W_{L}^{+} W_{L}^{-}$ 

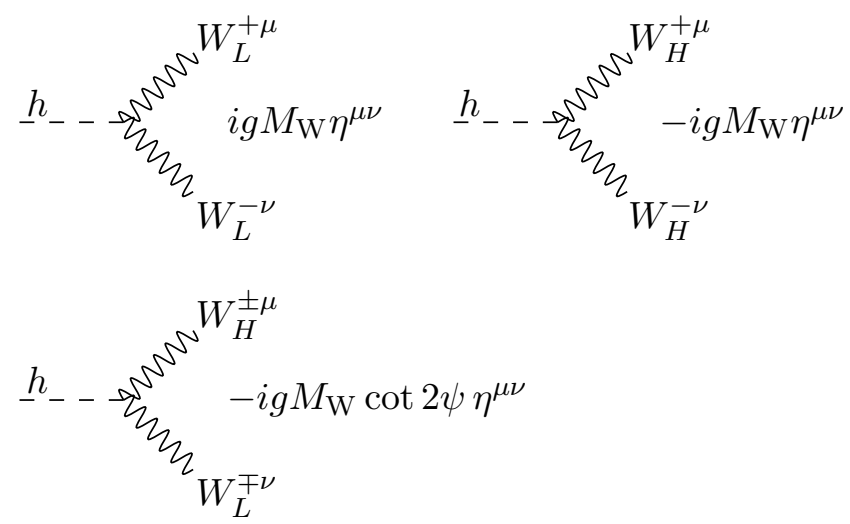

Figure 2: Cubic couplings between the physical Higgs boson $h$ and pairs of charged gauge bosons.

and $h W_{H}^{+} W_{H}^{-}$. These cancellations can be traced back to Eq. (10), and are therefore directly related to the crucial cancellation of quadratic divergences. Measuring the diagonal couplings would provide the most direct way to verify the little Higgs model. Unfortunately, experimentally this is a difficult task: the measurement requires associated production of a $W_{H}$ boson with a Higgs. The cross section for this process is very small: with $100 \mathrm{fb}^{-1}$ integrated luminosity at the LHC there are typically only tens of events in this channel, rendering it virtually unobservable once specific final states are considered. It is much easier to measure the off-diagonal couplings, such as $h W_{H}^{3} Z$ and $h W_{H}^{ \pm} W_{L}^{\mp}$. Although these couplings do not directly participate in the cancellation of quadratic divergences, verifying their structure would provide a strong evidence for the crucial feature of the model, Eq. (9). Indeed, the factor of $\cot 2 \psi$ in these couplings is a unique consequence of Eq. (9). To illustrate this point, consider an alternative "big Higgs" theory with the same $[S U(2) \times U(1)]^{2}$ gauge structure as the little Higgs, but with a Higgs field transforming only under a single $S U(2) \times U(1)$ factor with $\mathrm{SM}$ quantum numbers. (The one-loop quadratic divergence in the Higgs mass parameter is not canceled in this theory.) This theory predicts $h W_{H}^{3} Z$ and $h W_{H}^{ \pm} W_{L}^{\mp}$ vertices of the same form as in the little Higgs model, but with the replacement $\cot 2 \psi \rightarrow \cot \psi$. Thus, if the mixing angle $\psi$ can be obtained independently, the measurement of these vertices would act as a discriminator 
between the little Higgs and the alternative theory.

\section{Production and decay of heavy gauge bosons}

Heavy gauge bosons $W_{H}^{a}$ and $B_{H}$ are produced at the LHC predominantly through their coupling to quarks. The charges of the SM fermions under the extended $[S U(2) \times U(1)]^{2}$ gauge group are constrained by the requirement that they have correct transformation properties under its low-energy subgroup. We choose the left-handed fermions to transform as doublets under $S U(2)_{1}$ and singlets under $S U(2)_{2}$; the right-handed fermions are singlets under both $S U(2)$ 's 4 . The couplings of the heavy $S U(2)$ bosons have the form

$$
g \cot \psi W_{H \mu}^{a}\left(\bar{L} \gamma^{\mu} \frac{\sigma^{a}}{2} L+\bar{Q} \gamma^{\mu} \frac{\sigma^{a}}{2} Q\right),
$$

where $L$ and $Q$ are the left-handed lepton and quark fields, and we suppress the generation indices. The charges of the fermions under the two $U(1)$ groups have to add up to the SM hypercharge, $Q_{1}+Q_{2}=Y$, but are otherwise unconstrained. This implies a high degree of model-dependence in the couplings of the $B_{H}$ boson to fermions. Note that the strongest electroweak precision constraints on the little Higgs model [3, 5] arise precisely from the diagrams involving the $B_{H}$. Certain choices of the fermion charges and the angle $\psi^{\prime}$ can help minimize these constraints [6]; alternately, the extra $U(1)$ can be eliminated completely [8] without introducing significant fine tuning due to the smallness of the coupling $g^{\prime}$. Given this model uncertainty in the $U(1)$ sector, we will concentrate on the production and decay of the $S U(2)$ heavy bosons.

In $p p$ collisions at the LHC, the heavy gauge bosons are predominantly produced through $q \bar{q}$ annihilation. The sub-process $q g \rightarrow W_{H} q$ is considerably smaller and could be separately identified due to the presence of a high $p_{T}$ jet. Fig. 3 shows the leading order production cross section of $W_{H}$ as a function of its mass, for the case $\psi=\pi / 4$. The general case may be obtained from Fig. 3 by simply scaling by $\cot ^{2} \psi$. Two-body decay channels of the $W_{H}^{3}$ include $W_{H}^{3} \rightarrow \bar{f} f$, where $f$ is any of the SM quarks or leptons, as well as $W_{H}^{3} \rightarrow Z h$. (The decay $W_{H}^{3} \rightarrow B_{H} h$ may also be allowed in certain regions of the parameter space.) Partial decay widths in all these channels are easily 


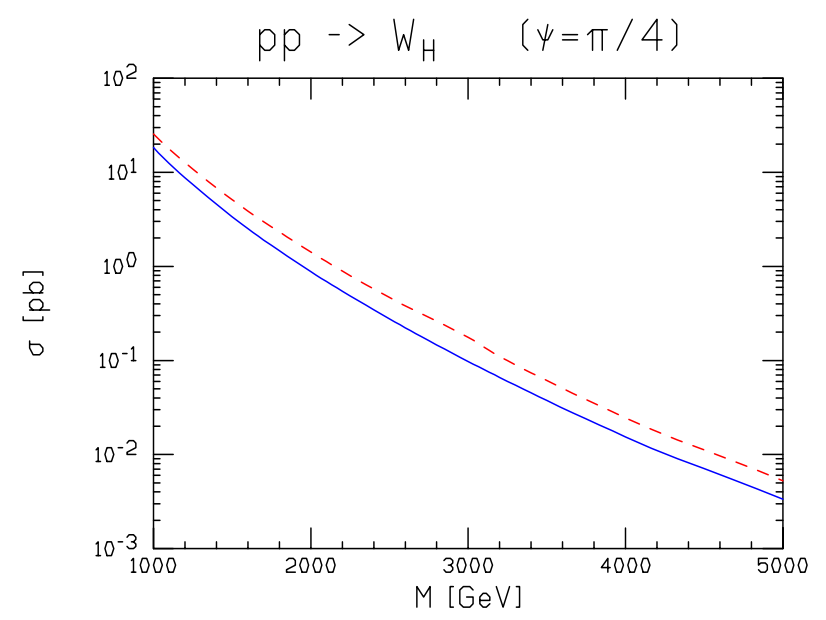

Figure 3: Production cross sections for $W_{H}^{3}$ (solid) and $W_{H}^{ \pm}$(dashed) at the LHC, for $\psi=\pi / 4$. We use the CTEQ5L parton distribution function.

computed using the Feynman rules in Fig. 1:

$$
\begin{aligned}
\Gamma\left(W_{H}^{3} \rightarrow \bar{\ell} \ell\right) & =\frac{g^{2} \cot ^{2} \psi}{96 \pi} M, \\
\Gamma\left(W_{H}^{3} \rightarrow \bar{q} q\right) & =\frac{g^{2} \cot ^{2} \psi}{32 \pi} M, \\
\Gamma\left(W_{H}^{3} \rightarrow Z h\right) & =\frac{g^{2} \cot ^{2} 2 \psi}{192 \pi} M,
\end{aligned}
$$

where $M=g f / \sin 2 \psi$ is the mass of the $W_{H}$ triplet, and we neglect corrections of order $\epsilon=v / f$ (including the effects of non-zero top mass). Summing over all the quark and lepton channels and ignoring the $B_{H} h$ mode (and all $N$-particle final states with $N \geq 3$ ) results in a total width

$$
\Gamma_{\text {tot }}=\frac{g^{2}}{192 \pi}\left(\cot ^{2} 2 \psi+48 \cot ^{2} \psi\right) M
$$

Partial decay widths of the $W_{H}^{ \pm}$bosons are easily obtained from (13) using the isospin symmetry, which is accurate to leading order in $\epsilon$.

\section{Testing the model at the LHC}

The discovery reach of the LHC for the $W_{H}^{3}$ and $W_{H}^{ \pm}$gauge bosons is quite high. The cleanest mode is $W_{H}^{3} \rightarrow \ell^{+} \ell^{-}$, with $\ell=e$ or $\mu$. Existing $Z^{\prime}$ studies 
at the LHC [7] indicate that these channels are virtually free of backgrounds. If we establish the discovery reach requiring the observation of 10 events, than for $100 \mathrm{fb}^{-1}$ luminosity this translates into a mass reach of approximately $M=5 \mathrm{TeV}$ for $\psi=\pi / 4$. Depending on the structure of the $U(1)$ sector, electroweak precision constraints on the model can be satisfied for $f$ as low as $2 \mathrm{TeV}$ [5, 9], corresponding to $M \sin 2 \psi \geq 1.3 \mathrm{TeV}$. Thus, there is a wide range of allowed parameters for which the extra gauge bosons are observable.

Discovering the $W_{H}$ triplet does not by itself provide a striking signature for the little Higgs model. Indeed, one can imagine many alternative theories in which such a triplet is present. As we already mentioned, a good way to distinguish between the little Higgs and alternative theories is to independently measure the mixing angle $\psi$ and the magnitude of the $h W_{H}^{3} Z$ and $h W_{H}^{ \pm} W_{L}^{\mp}$ couplings. Let us outline how these measurements can be performed at the LHC.

The number of events in the $p p \rightarrow W_{H}^{3} \rightarrow \ell^{+} \ell^{-}$channel, where $\ell=e$ or $\mu$, is given by

$$
N\left(\ell^{+} \ell^{-}\right)=\mathcal{L} \sigma_{\text {prod }}^{(0)} f\left(\tan ^{2} \psi\right)
$$

where $\mathcal{L}$ is the integrated luminosity, $\sigma_{\text {prod }}^{(0)}$ is the $W_{H}^{3}$ production cross section for $\psi=\pi / 4$ (see Fig. 3), and $f(x)=8 x^{-1}\left[192+(1-x)^{2}\right]^{-1}$. Assuming that $\sigma_{\text {prod }}^{(0)}$ can be accurately predicted, measuring the $\ell^{+} \ell^{-}$event rates yields $f(x)$, which can then be inverted to find the angle $\psi$ (up to a discrete ambiguity). If the $W_{H}$ triplet is not too heavy, the event rates are quite large, leading to small statistical uncertainties. For example, for $M=2.5 \mathrm{TeV}, \psi=\pi / 6$, and $\mathcal{L}=100 \mathrm{fb}^{-1}$, we find $N\left(e^{+} e^{-}\right) \approx 3500$, so the statistical uncertainty in measuring $\psi$ using this channel alone is less than $2 \%$. The number of $e^{+} e^{-}$ background events with such a high invariant mass is very small. The most important limiting factor in this measurement is the lack of precise knowledge of $\sigma_{\text {prod }}^{(0)}$, limited by the uncertainties in parton distribution functions. An alternative possibility is to measure the total width of the $W_{H}^{3}$ and use Eq. (14) to extract $\psi$; the uncertainty in this measurement is dominated by finite calorimeter resolution.

The number of events in each of the $p p \rightarrow W_{H}^{3} \rightarrow \bar{q} q$ channels is given by $3 N\left(\ell^{+} \ell^{-}\right)$. Verifying this relation provides a good test of the universality of the $W_{H}^{3} \bar{f} f$ couplings, Eq. (12). Once the universality is confirmed, these channels can be combined to further reduce the statistical uncertainty in the $\psi$ determination.

Once the mixing angle $\psi$ is determined, one simply needs to count the 
number of events in the $p p \rightarrow W_{H}^{3} \rightarrow Z h$ channel. The little Higgs model predicts

$$
N(Z h)=\mathcal{L} \sigma_{\text {prod }}^{(0)} g\left(\tan ^{2} \psi\right),
$$

where $g(x)=(1-x)^{2} x^{-1}\left[192+(1-x)^{2}\right]^{-1}$. Taking again the sample parameter values, $M=2.5 \mathrm{TeV}, \psi=\pi / 6$, and $\mathcal{L}=100 \mathrm{fb}^{-1}$, we obtain $N(Z h) \approx 200$. The alternative "big Higgs" theory predicts $N(Z h)=0.5 N\left(l^{+} l^{-}\right)$, or about 10 times more $Z h$ events for the sample parameter values than in the little Higgs model. (We assume that the fermion couplings to the gauge bosons of the "big Higgs" model are identical to Eq. (12).)

In order to evaluate the observability of this mode, we have assumed that the Higgs boson is heavy enough to decay into a $W$ pair and considered the process [10]

$$
W_{H}^{3} \rightarrow Z h \rightarrow Z W^{+} W^{-} \rightarrow 4 j+e \nu .
$$

We use the package MadGraph [11] to estimate the corresponding backgrounds. We require that the kinematics of the observed final state be consistent with the decay chain (17). (Note that both the $W_{H}^{3}$ mass and the Higgs boson mass will be known fairly well by the time this experiment becomes feasible.) We find that this requirement reduces the background to manageable levels, allowing one to clearly distinguish between the little Higgs model with the sample parameter values and the "big Higgs" alternative with $100 \mathrm{fb}^{-1}$ integrated luminosity.

While in the above discussion we concentrated on the $W_{H}^{3}$ boson, the same measurements can be performed using its charged partners $W_{H}^{ \pm}$. Once the isospin structure of the couplings is verified, the measurements in $W_{H}^{3}$ and $W_{H}^{ \pm}$channels can be combined to further improve the statistics.

\section{Conclusions}

If the little Higgs model is a part of the solution to the hierarchy problem, the extra gauge bosons present in this model should be light enough to be copiously produced at the LHC. The model makes definite predictions for the couplings of these gauge bosons to the light SM Higgs boson, which can be measured at the LHC in decays such as $W_{H}^{3} \rightarrow Z h$ and $W_{H}^{ \pm} \rightarrow W_{L}^{ \pm} h$. This measurement tests the detailed structure of the Higgs sector of the model, and can be used to distinguish it from alternative theories with the same gauge group. 


\section{Acknowledgments}

We would like to thank Nima Arkani-Hamed, JoAnne Hewett, Ian Hinchliffe, Hitoshi Murayama, Michael Peskin, Tom Rizzo, Tim Stelzer, John Terning and Jay Wacker for useful discussions related to this work. G. B. and M. P. are supported by the Director, Office of Science, Office of High Energy and Nuclear Physics, of the U. S. Department of Energy under Contract DEAC03-76SF00098. A. P. is supported by the U. S. Department of Energy under Contract DE-AC03-76SF00515.

\section{References}

[1] N. Arkani-Hamed, A. G. Cohen, E. Katz and A. E. Nelson, JHEP 0207, 034 (2002) [arXiv:hep-ph/0206021].

[2] N. Arkani-Hamed, A. G. Cohen, E. Katz, A. E. Nelson, T. Gregoire and J. G. Wacker, JHEP 0208, 021 (2002) [arXiv:hep-ph/0206020].

[3] C. Csaki, J. Hubisz, G. D. Kribs, P. Meade and J. Terning, arXiv:hep$\mathrm{ph} / 0211124$.

[4] Another simple and consistent $S U(2)$ charge assignment corresponds to interchanging $S U(2)_{1} \leftrightarrow S U(2)_{2}$; the results for this case can be obtained by exchanging $\psi \leftrightarrow \pi / 2-\psi$ in Eq. (12) and below.

[5] J. L. Hewett, F. J. Petriello and T. G. Rizzo, arXiv:hep-ph/0211218.

[6] J. Terning, private communication.

[7] "ATLAS detector and physics performance. Technical design report. Vol. 2," CERN-LHCC-99-15.

[8] N. Arkani-Hamed, private communication.

[9] The direct Tevatron bounds of Ref. [5] are avoided, for example, in models where only a single $U(1)$ is gauged.

[10] An alternative decay chain, $W_{H}^{3} \rightarrow Z h \rightarrow \ell^{+} \ell^{-}+4 j$, has a branching ratio smaller than (17) by about a factor of 3, but could also be useful, especially for relatively light $W_{H}^{3}$. For a lighter Higgs, one could use the decay chain $W_{H}^{3} \rightarrow Z h \rightarrow \ell^{+} \ell^{-} b \bar{b}$. 
[11] T. Stelzer and W. F. Long, Comput. Phys. Commun. 81, 357 (1994) [arXiv:hep-ph/9401258]; F. Maltoni and T. Stelzer, [arXiv:hepph/0208156]. See also http://madgraph.physics.uiuc.edu/. 